\title{
Hacia una cultura plurilingüe: prácticas educativas en contextos migratorios
}

Daniela Carpani

\section{(2) OpenEdition \\ 12 Journals}

Edición electrónica

URL: http://journals.openedition.org/esp/935

DOI: $10.4000 /$ esp.935

ISSN: 2532-0319

Editor

Centre d'Information sur l'Éducation Bilingue et Plurilingue

Edición impresa

Fecha de publicación: 1 diciembre 2016

Paginación: 43-56

ISSN: 1127-266X

\section{Referencia electrónica}

Daniela Carpani, « Hacia una cultura plurilingüe: prácticas educativas en contextos migratorios »,

Éducation et sociétés plurilingues [En línea], 41 | 2016, Puesto en línea el 20 octubre 2017, consultado el 20 abril 2019. URL : http://journals.openedition.org/esp/935 ; DOI : 10.4000/esp.935 


\section{HACIA UNA CULTURA PLURILINGÜE: PRÁCTICAS EDUCATIVAS EN CONTEXTOS MIGRATORIOS}

\section{Daniela GarPani}

L'articolo è il punto di partenza di un progetto di ricerca-azione intitolato "Il plurilinguismo come ricorso educativo, cognitivo e sociale" introdotto in una classe pilota dell'insegnamento primario dotata di una didattica curricolare interculturale. I due anni di pratica e d'osservazione conclusisi recentemente (giugno 2015) ci offrono un'occasione di riflessione sul potenziale di pratiche rivolte alla formazione per un'educazione plurilingue intesa non solo come valore, ma anche come competenza.

Parole chiave: ricerca-azione; plurilinguismo; classe polota; curriculum interculturale; educazione plurilingue

L'article est le point de départ d'un projet de recherche-action intitule "Le plurilinguisme comme recours éducatif, cognitif et social", implanté dans une classe pilote du primaire dotée d'un curriculum interculturel. Les deux années de pratique et d'observation qui se sont terminées récemment (juin 2015) nous offrent l'occasion de réfléchir au potentiel de pratiques tournées vers la formation à une éducation plurilingue, comprise non seulement comme une valeur mais également comme une compétence.

Mots-clés: recherche-action; plurilinguisme; classe pilote, curriculum interculturel; éducation plurilingue

The article describes the action-research called "Plurilingualism as a pedagogical, cognitive and social resort" that was applied in an experimental class of a primary school, using an intercultural curriculum. Two years of actual practice and observation (until fune 2015) allow us to think out the potential connected to plurilingual training and education, considered to be not only a value but also a skill.

Key-words: action-research; plurilingualism; experimental class, intercultural curriculum; plurilingual education

EL CONTEXTO
E l último Dossier sobre la inmigración evidencia que, entre 2007 y 2014, el contingente de alumnos alófonos nacidos en Italia ha aumentado del $12,4 \%$ frente al crecimiento del $5,2 \%$ de la totalidad de menores extranjeros presentes en el territorio. Una inversión de tendencia que empieza en el año escolar 2013/2014 y que se percibe sobre todo en la escuela primaria que es, actualmente, el grado que más acoge a este contingente con su 10,6\% de presencias sobre el total de aprendices (Dossier 2015: 206-207). 
Hacia una cultura plurilingüe: prácticas educativas en contextos migratorios

D. CARPANI
Este cambio, tal vez por ser bastante reciente, no estimula una reflexión sobre las exigencias de un público nuevo ni valora las potencialidades que su presencia puede significar. En efecto, en el marco de las políticas de integración observadas por el reciente Migrant intergration Policy Index de 2015, es justamente la educación nuestra asignatura pendiente colocándose, a diferencia de otros aspectos analizados, en el range de "slight unfavourable". Más específicamente, tal como había ocurrido en la versión de 2013, la dimensión de las "nuevas oportunidades" se vuelve a considerar el punto débil del sistema educativo italiano: "Schools in IT are rarely used as a common space for social integration, with measures on 'new opportunities' scoring only 10/100 and ranking $25^{\text {th}}$; the main guidance for schools, is to use cultural mediators to involve immigrant parents in schools and school-based IT courses; IT pupils are not encouraged to open up to immigrant peers, their languages and their cultures; Immigrant languages are absent from the curriculum, unlike in the majority of countries (e.g. AT, FR, PT, ES, CH); Following abolition of the 30\% quota on foreign pupils in classrooms, no new policy promotes mixed schools/classrooms or diversity in the teaching force." http://www.mipex.eu/

La experiencia que aquí se presenta pretende dar constancia de un intento de flexibilización y apertura del currículo en la dirección indicada no solo en el documento que acabo de citar, sino también en las recomendaciones del Consejo Europeo a propósito de la educación plurilingüe entendida como competencia imprescindible para las nuevas generaciones de estudiantes del mundo globalizado.

Se trata de una investigación-acción fruto de una experiencia de colaboración entre universidad y escuela, iniciada en una clase de primaria con currículo piloto intercultural de un instituto genovés (http://www.comprensivocornigliano.it/) - ya sede de mis investigaciones a partir de 2010 - que acoge un significativo número de familias de origen extranjero y por eso pululante de las llamadas "lenguas inmigradas" - (Vedovelli 2004), fruto, estas últimas, de asentamientos estables que han modificado la incidencia de alófonos en el sistema educativo y, sin embargo, ausentes en las aulas no solo por razones meramente práctico-organizativas, sino también por el estigma social que les atribuye la sociedad de acogida que distingue, en términos de valoración, el estudio de una lengua extranjera de las prácticas lingüísticas asociadas a los inmigrantes. Un estigma que comparten las mismas familias alófonas, a menudo rehenes de una autopercepción negativa de la propia cultura y, 
Hacia una cultura plurilingüe: prácticas educativas en contextos migratorios

D. CARPANI

DOCENTES, FAMI LIAS Y ALUMNOS: EL ROL DE LA LENGUA MATERNA por lo tanto, de su código de pertenencia (Caravedo 2010; Ariolfo 2012).

Se trata de actitudes y representaciones sociales que cumplen un rol fundamental en el mantenimiento del bagaje cultural de los alumnos de origen extranjero (Cavalli et al 2003), quienes, a pesar de su joven edad que debería precaverlos contra este tipo de estereotipos, corren el riesgo de encaminarse hacia un bilingüismo sustractivo si la escuela no encuentra formas para valorar no tanto una lengua en particular sino más bien una educación inclusiva, basada en la apertura y en el intercambio productivo de todos los códigos en contacto, que repercuta positivamente y transversalmente en el conjunto del grupo clase, estudiantes autóctonos incluidos.

Basándome en los resultados de investigaciones de campo que confirmaban la problematicidad de la gestión educativa en situaciones de contacto lingüístico por migración, (Autor en prensa: 2010), decidí poner en práctica un recorrido de enseñanza plurilingüe basado en la movilización de conocimientos individuales y de grupo partiendo de las lenguas presentes en el aula que fue, durante dos años, el escenario de la investigación-acción cuyos efectos, sobre todo en términos de construcción de la conciencia lingüística, se van a presentar a continuación.

Los docentes, sobre todo en la educación primaria, no pasan por alto las recomendaciones a la valorización de la diversidad citada en el último documento del Ministero dell'Istruzione, Università e Ricerca (MIUR) dedicado a la escuela plural (Le linee guida per l'accoglienza e l'integrazione degli alunni stranieri, 2014: 18-19) (http://hubmiur.pubblica.istruzione.it/web/ministero/focus 1902 14) y trabajan diariamente, tal y como pudimos comprobar, en valores como la aceptación, el respeto y la tolerancia. Los carteles multilingües, los documentos para la primera acogida en idiomas distintos, los mediadores culturales ya no son una excepción, y atestiguan un importante cambio de perspectiva cultural. En el día a día, sin embargo, no se da la deseada "renovación didáctica a favor de todos" (ibi) por cierto apego al enfoque integracionista de la primera hora -a raíz de la llegada de estudiantes alófonos de los años 90- focalizado sobre todo en el aprendizaje del italiano. Y esto a pesar de las profundas diferencias que separan los menores de aquel entonces, hijos de la reagrupación y, en su mayoría, con experiencias previas de escolarización en el país de origen (Autor en prensa: 2008) de los "nuevos italianos" de hoy, alfabetizados en la educación infantil italiana, que entran en la escuela primaria, 
Hacia una cultura plurilingüe: prácticas educativas en contextos migratorios

D. CARPANI como veremos, hablando exactamente como sus compañeros autóctonos.

Las familias, por su parte, en un contexto, reitero, muy peculiar de contacto lingüístico, no saben qué código usar con sus hijos. De no ser que exista una clara voluntad autónoma de mantenimiento de la lengua de origen, fomentada por cursos impartidos fuera de la escuela, como suele pasar sobre todo entre usuarios de lenguas no románicas. Modélico es el caso de los sinohablantes sólidamente aferrados a su LM a los que se unen, por lo que pudimos comprobar en el colegio sede de nuestra experiencia, los aprendices arabófonos y, en la clase objeto de mis análisis, una alumna tamilhablante.

Una "fidelidad" al código familiar que no se da, por el contrario, en las familias procedentes de América Latina. Las investigaciones de los últimos diez años, focalizadas sobre este contingente, han evidenciado una clara tendencia por parte de los adultos a guardar con su lengua una relación de doble diglosia (Ariolfo 2012: 19-20) no solo hacia el italiano (interlingǘstica) sino también hacia la variante peninsular (intralingüística) percibida - por tratarse de un "español preciso" (Calvi 2015: 15) - como más prestigiosa que su propia variante.

A esto hay que añadir, debido a la cercanía morfológica de los dos idiomas, cierta aceptación fatalista de un mixed code entre español e italiano (Bonomi 2010) que conlleva indefectiblemente consecuencias negativas en las nuevas generaciones. Un reciente análisis llevado a cabo con alumnos de primaria de un colegio genovés ha demostrado que, estadísticamente, los niños hispanohablantes son los que más se equivocan en la ortografia italiana a pesar de haber nacido aquí y de haber sido incorporados en las estructuras educativas en edad temprana. Se trata, sobre todo, de errores fonológicos resultado de erradicados y generalizados hábitos lingüísticos de esta comunidad que tiende a mezclar palabras, estructuras sintácticas o sonidos de los dos códigos en contacto, dificultando una clara distinción entre ambos por parte de sus hijos. Un fenómeno muy bien conocido por los estudiosos de lingüística española que desde hace años nos ocupamos del llamado bilingüismo social que, como recuerda Maria Vittoria Calvi, "a diferencia de lo que ocurre en las situaciones de bilingüismo más estable, hace que los procesos de variación del repertorio de los recién llegados se combinen con los comportamientos de los bilingües que ya han adquirido las competencias lingüísticas más avanzadas en la nueva lengua, lo cual dificulta la tarea de distinguir entre interferencias, préstamos y CC [cambio de código]" (Calvi 2015: 6). 
Hacia una cultura plurilingüe: prácticas educativas en contextos migratorios

D. CARPANI

\section{LA INVESTIGACIÓN-} ACGIÓN: UN

PROCESO COMPARTIDO
En la convicción de que las actitudes negativas, las incertidumbres didácticas y los estereotipos no se pueden resolver "desde fuera" sino más bien a través de un actuar compartido, se ha abogado por una metodología que promete favorecer, además de los progresos teóricos, mejores acciones educativas durante la investigación y después de la investigación misma, gracias a la colaboración de los sujetos involucrados.

La maestra con la que trabajé, que según lo que ya había podido comprobar durante precedentes colaboraciones, cabía perfectamente en el perfil de "innovatori naturali", citados en el reciente decreto aprobado en julio de 2015 por el Gobierno italiano ( $L a$ Buona Scuola: 47) (http://labuonascuola.gov.it/documenti/lbs_web.pdf?v=0b45ec8 ) por haber dado muestra de querer explotar constructivamente la potencial riqueza dada por el contacto entre niños de orígenes distintos. Suyo es el diseño de un curso piloto (activado en 2008) basado en la interculturalidad y en la inclusión cuya reedición iba a empezar en el año en que presenté el proyecto de investigaciónacción Il plurilinguismo come risorsa cognitiva e sociale 2013/2015 (El plurilingüismo como recurso cognitivo y social) inspirado en las indicaciones de la Guide pour le développement et la mise en cuvre de curriculums pour une éducation plurilingue et interculturelle y, más en general, en la teoría del language awarness ya experimentado con éxito en países limítrofes con significativa presencia de alumnos extranjeros de distintos orígenes (Auger 2013). La observación fue llevada a cabo a través de notas de campo redactadas por mí o por la maestra y codificadas posteriormente según procesos deductivos o inductivos sugeridos por las circunstancias.

En esta ocasión se presentarán algunas reflexiones focalizadas en la formación de la conciencia lingüística, por considerarse el primer peldaño de un proceso de aprendizaje basado en el ser, el hacer y el aprender (García, Wei 2014: 80). En la parte conclusiva, se dará constancia de algunas actividades de reflexión metalingüística que caracterizaron el segundo año de curso y de los primeros efectos del proyecto en términos de espiral de acción compartida.

Antes de empezar las actividades (octubre de 2013) redacté un primer cuadro de la situación lingüística de las familias a través de un cuestionario rellenado por los padres durante una reunión preliminar organizada por la maestra. Un encuentro en el que se ilustraron los principios del proyecto recalcando la importancia de hablar (o seguir hablando) con los pequeños las lenguas de origen 
Hacia una cultura plurilingüe: prácticas educativas en contextos migratorios

D. CARPANI con el fin de cerciorar a los presentes de que dicha elección no dificultaría el aprendizaje del italiano. Además aclaramos que, en nuestro caso, era una suerte de conditio sine qua non del recorrido que íbamos a seguir, focalizado en el fomento de la conciencia plurilingüe y en la observación de su efectos, a través de hábitos de transferencias intra e interlingüísticas y en términos de capacidad de reflexión metalingüística y de intercomprensión. Objetivos que las muchas lenguas representadas en la clase -albanés, hindi punjabi, tamil, armenio, árabe, romaní, español, italiano por un total de catorce alófonos sobre veintiuno- prometían favorecer. El paso siguiente fue cruzar los datos relativos a las informaciones de las familias con las de sus hijos, a través de una encuesta sobre usos lingüísticos y actitudes hacia las lenguas redactadas esta vez por los alumnos (1). Por aquel entonces (octubre de 2013), entre los aprendices de origen extranjero, solo tres de reciente inmigración (lengua materna español, armenio y árabe) tenían dificultades de producción y comprensión en italiano en los dos primeros casos y de producción en el tercero. Sin embargo, el alumno arabófono (mayor de un año respecto a sus compañeros) que declaraba hablar también inglés en casa, era capaz de movilizar autónomamente sus recursos para superar las dificultades comunicativas, gracias a una conciencia muy clara de la importancia de conocer cuantas más lenguas extranjeras posibles, fomentada y asesorada por el padre, maestro de inglés en Egipto, su lugar de origen. Lo que había generado en el hijo una capacidad de "indipendent translanguaging pattern" (García,Wei 2014: 87) mucho más desarrollada que la de sus compañeros. Los demás, como se decía, en su totalidad nacidos en Génova, dominaban perfectamente la lengua del país de acogida.

Las respuestas a la pregunta "¿Qué lenguas hablas en casa/qué lengua hablan tus padres entre sí?" oscilaban entre incoherencia y, al parecer, el deseo de dar una contestación "adecuada" a las expectativas del investigador. Así un niño que declaraba expresarse en árabe y en italiano afirmaba que sus padres se comunicaban en italiano (situación improbable siendo ambos arabófonos); el pequeño armenio, que apenas lograba hacerse entender en la nueva lengua, contestaba que toda la familia hablaba en italiano a pesar de que la madre se expresara solo en armenio, como habíamos podido comprobar durante la reunión preliminar. Otra alumna (hija de una pareja mixta con padre hindihablante) sacaba de la chistera un improbable inglés y francés para la comunicación familiar, y tres alumnos con ambos padres extranjeros, elegían el italiano como lengua del hogar. 
Hacia una cultura plurilingüe: prácticas educativas en contextos migratorios

D. CARPANI

\section{EL PRIMER} PORTFOLIO DE LAS LENGUAS
Por lo que se refiere a las lenguas de origen de los compañeros tampoco faltaron sorpresas sobre todo con las percibidas como más "exóticas" (tamil, hindi punjabi y armenio) que se atribuyeron de forma más bien casual. Lo que sugirió una puesta en común de los resultados y una discusión que nos permitió tener un cuadro lo suficientemente claro de la situación lingüística de la clase.

Una vez sentadas las bases de un conocimiento mutuo y empezadas las primeras actividades de apoyo en español, árabe y francés desarrolladas por los mediadores lingüísticos y por una maestra francesa de intercambio asignada al curso piloto, tratamos de despertar y movilizar las respectivas lenguas de origen que empezaban a aflorar: el simple hecho de descubrir un abanico de sonidos tan distintos entre sí estimulaba la natural curiosidad infantil favoreciendo el contacto y el intercambio de conocimientos de todo el grupo-clase, italohablantes incluidos. Posteriormente, a través de la comparación léxica, se estimuló a los niños a detectar semejanzas entre lenguas cercanas o muy distantes tipológicamente, sentando las bases para un aprendizaje colaborativo en el que cada uno podía aportar su grano de arena. Esta primera etapa (diciembre de 2013) se concluyó con la redacción de su primera biografía lingüística.

En una versión muy simplificada del Portfolio se volvían a proponer algunos interrogantes del cuestionario inicial integrados por otros (1 En casa hablo en; 2 ¿Qué lenguas hablan las maestras y los maestros? 3 ¿Cuántas palabras sé en francés, inglés, español, árabe?; 4 ¿Qué sé hacer en francés, inglés, español, árabe (hablar/cantar/leer/escribir); 5 Yo y las lenguas de la clase; 6 ¿Te gustan las lenguas que aprendes en el cole?; 7 ¿Por qué?) para observar los eventuales cambios en la conciencia y en la actitud de los informantes y obtener un primer feedback de las actividades de plurilingüismo. Las dos últimas respuestas fueron redactadas con la ayuda de las maestras, que facilitaron la escritura.

Ya a partir de las elecciones relativas a la primera pregunta se apreciaba una mayor "pertinencia" con la situación familiar (si se excluye un "indiano e italiano" de un alumno italiano y un "francés y árabe" del alumno egipcio). La mayoría de los niños extranjeros declaraba conocer "muchas" palabras en la lengua del hogar (algunos atreviéndose a cuantificarlas disparando números al azar: de 1000 pasando por 102, 10, 12, etc.).

Por lo que se refiere a la apreciación (pregunta 5), el inglés, tal vez por ser percibido materia de estudio con dos horas curriculares, se 
Hacia una cultura plurilingüe: prácticas educativas en contextos migratorios

D. CARPANI colocaba en posición inferior al español. Un primado debido probablemente a los ocho compañeros hispanohablantes y a la masiva presencia de esta minoría en el barrio de Cornigliano, considerado por esta razón una de las zonas "latinas" por antonomasia de Génova. El francés, por su parte, oscilaba entre apreciación, indiferencia o rechazo. Estas elecciones parecían evidenciar una fuerte relación no tanto con el prestigio de una u otra lengua, concepto todavía ajeno a los niños, sino más bien con su visibilidad. Según este principio, el armenio, por ejemplo, el más "ausente" tanto por las objetivas dificultades de encontrar recursos a nuestro alcance, como por la actitud del niño que, a pesar de hablarla en familia, afirmaba "no saberla", resultaba ser la lengua menos "simpática", seguida por el hindi punjabi (no olvidemos que las lenguas del hogar indicadas a principio de año por la niña habían sido inglés y francés). El tamil, en cambio, exhibido orgullosamente por la alumna cingalesa que solía intervenir espontáneamente traduciendo palabras o frases para que la clase supiera cómo se decía en su país, se colocaba en buena posición, junto con el árabe que, como ya se ha especificado, contaba, igual que el español y el francés, con actividades de apoyo hechas por nativos.

En cuanto a las actividades de plurilingüismo, todos demostraban apreciarlas. Entre las respuestas abiertas a las preguntas 6 y 7 la mayoría sacaba a colación motivos genéricamente instrumentales ("Aprendo las lenguas porque de mayor sabré las lenguas"; "Porque las aprendo"; "Porque sé unas cuantas palabritas"). Solo cuatro informantes (dos hispanohablantes, la alumna albanesa y uno de los tres arabófonos presentes) citaban de forma explícita su LM escribiendo respectivamente: "Porque aprendo mi lengua, las palabras y a leer"; "Porque puedo hablar en mi idioma"; "Porque es mi lengua"; "Me gusta la lengua árabe".

En la segunda versión del Portfolio, realizada en abril del mismo año para seguir observando la evolución de los aprendices, la sección dedicada al interés hacia las lenguas (anteriormente representada con emoticonos) fue sustituida por dos preguntas a respuesta abierta ("¿Qué idioma despierta más tu curiosidad?"; “PPor qué?”; “¿Te gustaría aprender una lengua más?”; “CGuál?”; “¿Por qué?") que los alumnos pudieron redactar autónomamente gracias a la competencia de lectoescritura ya adquirida. Entre los 16 participantes, 4 contestaron negativamente a ambas preguntas con motivaciones distintas: "No porque no"; "No porque es aburrido"; "No porque son muy difíciles" y "No porque no me interesa". Un alumno se sentía atraído por el japonés pero, sin 
Hacia una cultura plurilingüe: prácticas educativas en contextos migratorios

D. CARPANI
¿DÓNDE ESTÁ MI LENGUA? embargo, no le hubiera gustado aprenderlo por considerarlo "dificil".

Todos los que especificaron qué lengua les atraía más eligieron una hablada por algún compañero (incluidos hindi punjabi y armenio antes "invisibles"). Entre las motivaciones, seis sobre nueve eligieron razones pragmáticas ("Porque me gusta aprender una lengua que no sabes"; "Sí porque aprender otras lenguas sirve para aprender bien muchas lenguas"; "Quisiera aprender árabe porque me gustaría hablarlo"; "Sí porque así cuando vamos de vacaciones a algún lugar la podemos hablar"; "Sí porque quiero aprender español porque es una lengua fácil es como el italiano y así lo aprendemos"; "El armenio porque no se entiende nada de nada"). Nadie aludió a simpatías o antipatías hacia los compañeros para explicar las razones de la elección.

Se trataba de una actitud reflexivo/racional a través de la cual los aprendices parecían haber interiorizado datos y principios reiteradamente explicitados (la necesidad de saber comunicar con personas que no hablan la misma lengua, la productividad de la comparación entre códigos distintos, el respeto hacia la cultura de los compañeros). Lo que favorecía la conciencia de formar parte de un grupo en el cual la diversidad lingüística constituía un valor añadido útil para el aprendizaje en general y, más concretamente, rentable para la vida fuera de las paredes del colegio. Un futuro que los alumnos imaginaban, con razón, a imagen y semejanza de su pequeño microcosmos plurilingüe.

Una vez creadas las bases para un investment a través del cual los aprendientes expresaban el deseo de enriquecer su capital cultural decidimos comparar sus convicciones abriendo una ventana sobre la dimensión afectiva, espontánea e íntima de la relación de los pequeños con sus vivencias lingüísticas.

Casi a finales del año, en el mes de mayo, se les pidió que hicieran un autorretrato especificando en qué parte del cuerpo colocaban la o las lenguas presentes en su vida en el modelo de propuestas ya experimentadas entre alumnos de la misma edad (Favaro 2013). La actividad fue acompañada por un comentario explicativo recogido individualmente y transcrito por la maestra.

Entre los 17 participantes los órganos mayoritariamente elegidos para la LM fueron el corazón seguido por la barriga, lugares donde se "guardaba" a menudo como un tesoro de mucho valor ("Porque papá quiere que lo hable y es precioso"; "Lo guardo en un lugar escondido porque es una lengua importante"; "En un lugar especial porque es la lengua de mi madre"). Interesantes los 
Hacia una cultura plurilingüe: prácticas educativas en contextos migratorios

D. CARPANI

\section{PRÁCTICAS DE EDUCACIÓN PLURILINGÜE}

comentarios de los dos alumnos que resultaban ser los más asesorados por la familia en el mantenimiento de la lengua del hogar (y del inglés). Ambos eligieron al cerebro como órgano de la LM motivando detalladamente sus elecciones: "El árabe porque está en el cerebro porque he nacido con esta lengua. El albanés y el francés está en los brazos porque estoy aprendiéndolos. El español está en la garganta porque estoy aprendiéndolo. El italiano en la barriga y en el corazón porque estoy contento de estar en Italia. El inglés está en mi rodilla porque es mi segundo idioma y me lo ha enseñado mi padre. El armenio está en la rodilla porque me lo ha enseñado David mientras juega)".

La niña, por su parte, comentaba: "El tamil está en el cerebro porque es mi lengua. El italiano en el cerebro porque lo aprendo en el colegio. El inglés en una pierna porque estoy aprendiéndolo y lo sé un poco. El albanés en el tobillo porque me gustaría aprenderlo. El armenio en una pierna porque querría aprenderlo. El árabe en la barriga porque conozco algunas palabras".

Los demás citaban, además de la LM, el francés, el español, el inglés, el armenio, el hindi y el albanés que colocaron mayoritariamente en las piernas seguidas por brazo, mano y garganta subrayando con las elecciones verbales la movilidad del proceso. A los muchos "Porque estoy aprendiéndola", otros añadieron "Estoy empezando a moverlo", "Porque la muevo", "Es como caminar", "Así la aprendo levantando la mano", "Porque con ella camino". Solo un aprendiz, muy a menudo ausente por problemas familiares, se limitó a nombrar los idiomas de los padres: "El italiano está en una pierna porque es un lugar seguro donde guardar la lengua de mi padre. El español está en el brazo; es un idioma precioso porque es el de mi madre".

A través de los dibujos pudimos comprobar cómo, a nivel espontáneo e íntimo, los alumnos asociaban una dimensión afectiva, estable, secreta representada por la LM con otra dinámica, móvil, in fieri de los idiomas que los rodeaban, y que estaban igualmente presentes en sus vidas.

Sentadas las bases para reforzar la conciencia lingüística, el segundo año fue dedicado a actividades basadas sobre todo en la comparación - encaminadas a la reflexión morfológica además de lexical - y en la intercomprensión.

Ya durante el primer año de curso se habían propuesto juegos para favorecer, lo más posible, la cooperación entre pares y el aprendizaje por descubrimiento basados tanto en las lenguas de la clase como en las presentes en el currículo (español, francés, 
Hacia una cultura plurilingüe: prácticas educativas en contextos migratorios

D. CARPANI inglés e italiano). Estas últimas - a través de comentarios inducidos sobre las características comunes del grupo de lenguas románicas, de las características fonéticas de cada idioma, de la relación entre fonema y grafema, etc. - constituyeron la ocasión para subrayar la productividad de estrategias de memorización entendidas como operación consciente para alcanzar objetivos de aprendizaje.

Si la elección de los posibles temas para desarrollar según una perspectiva plurilingüe solía ir en paralelo con el programa de las maestras, a menudo eran los comentarios de los aprendices los que nos indicaban el camino a seguir según los principios de una pedagogía "ground up". Sus comentarios sobre la facilidad/dificultad percibidas de los sonidos de las nuevas lenguas había llevado, por ejemplo, a la observación de la capacidad de mímesis fonética en francés, considerado por la mayoría como el idioma más difícil de pronunciar. Con el aporte de la maestra nativa que evaluó las performances individuales de los aprendices, pudimos detectar resultados significativamente superiores entre los 13 alófonos (sobre 18 participantes), quienes demostraron ser menos rehenes del filtro fonológico que los italohablantes.

El francés, además, lejos de desaparecer con la conclusión del periodo de estancia de la maestra (mayo de 2013), siguió siendo un válido recurso para las actividades del segundo año por cumplir con algunos importantes requisitos: no ser lengua madre de ninguno de los niños; ser una lengua extranjera y, sin embargo, no totalmente desconocida; no ser materia de estudio. Una suerte de "puente" alternativo al italiano para descubrir un potencial de bases transferenciales no solo léxicas, como se había hecho hasta aquel entonces, sino también sintácticas.

Así, la traducción al francés de las frases elegidas por los pequeños en la simulación de un encuentro con un nuevo amigo $(\dot{i} \mathrm{De}$ dónde eres?; ¿Cuántos años tienes?; ¿Qué lengua hablas?; ¿En qué curso estás?; ¿Qué te gusta?; ¿Quieres jugar a...?; ¿Quieres ser mi amigo?; ¿Quieres venir a mi casa?') y la comparación con el italiano permitió descubrir inductivamente -sobre todo gracias al aporte de cuatro alumnos, tres alófonos y una italiana - la obligatoriedad del sujeto en francés. Reflexión retomada y desarrollada sucesivamente por la maestra de inglés.

Estos reducidos ejemplos de los dos años de investigación-acción pretenden simplemente dar constancia de la posibilidad de transformar cualquier actividad en ocasión de reflexión lingüística a través del uso de todos los recursos útiles para mantener viva la curiosidad hacia sonidos, estructuras, palabras diferentes estimu- 
Hacia una cultura plurilingüe: prácticas educativas en contextos migratorios

D. CARPANI

\section{CONCLUSIONES}

lando la "spontaneus biliteracy" de los niños (García, Wei 2014: 61-62). En un sistema educativo como el italiano, aún aferrado, en esta etapa educativa, a un sustancial monolingüismo, solo muy relativamente paliado por la enseñanza del inglés, es sumamente importante "no tirar nada". No siempre es posible contar con mediadores, docentes de intercambio, investigadores que, como en el caso de la clase piloto, trabajen al lado de los docentes. Son estos últimos los que deben sacar provecho de los estímulos y de los conocimientos de sus alumnos moviendo los recursos que todos tenemos al alcance y transformándose en un aprendiente más. No es necesario ser políglotas para emprender el camino, como he demostrado reiteradamente a las maestras con las que he colaborado que, gracias a la toma de conciencia de las potencialidades del "pupils directed translanguaging" (García, Wei 2014: 81), han dado un paso más optando por una política de condivisión difusiva que constituye uno de los objetivos más importantes de la investigación-acción (Latorre 2003: 28). En la actualidad (2016), la clase piloto y la clase paralela con currículo tradicional (con un número aún mayor de estudiantes de origen extranjero) se reúnen para dedicar una hora semanal a actividades de plurilingüismo. Entre los efectos más evidentes de este círculo virtuoso, podemos destacar el despertar de las lenguas maternas entre los pequeños alumnos del curso paralelo estimulados y asesorados por los coetáneos de la clase piloto, más acostumbrados a compartir con los demás sus vivencias lingüísticas y, por eso, excelentes tutores de sus pares.

Los cambios que estamos experimentando, como efecto de una movilidad humana sin precedentes, han favorecido el nacimiento de nuevos enfoques en el campo de la educación lingüística. La escuela y, en particular, la educación primaria puede favorecer o dificultar el uso, el mantenimiento y el aprendizaje de una o más lenguas. La educación plurilingüe, entendida como competencia transversal basada en la movilización de recursos entre hablantes de idiomas diferentes, se ha revelado sumamente útil en contextos educativos caracterizados por una significativa presencia de alumnos procedentes de familias de migrantes. Se trata de una situación de contacto cultural y lingüístico muy peculiar, donde conviven estereotipos, expectativas y estigmas sociales que parece necesitar antes que nada un "enfoque translingǘstico" a través del cual se aprenda a usar y a valorar todos los recursos al alcance de los aprendices de forma dinámica, espontánea y consciente a la vez. Un proceso " (...) by which students and teachers engage in 
Hacia una cultura plurilingüe: prácticas educativas en contextos migratorios

D. Carpania

\section{BIBLIOGRAFÍA}

complex discursive practices that include ALL the language practices of ALL students in a class in order to develop new language practice and substain old ones, communicate and appropriate knowledge, and give voice to new sociopolitical realities by interrogating linguistic inequality" (García, Wei 2014: 66)

Su fuerza, como se decía, es despertar lenguas entre personas en carne y hueso que antes que nada tienen que reorientar sus categorías y convicciones en un espacio dinámico donde no haya ninguna competición entre distintos lenguajes de instrucción. Un reframing que debe involucrar a los docentes dispuestos a vivir su rol no solo como fuente de saberes, sino también de facilitadores y aprendientes a la vez para hacer aflorar, en términos cognitivos y de actitudes, las potencialidades de sus alumnos y también las propias. "All teachers in the 21 st century need to be prepared to be bilingual teachers (...); that is, they needs to see themselves as building and developing the student's additional languages while educating them. But in most teacher education institutions throughout the world, the student's multilingualism in an afterthought, and teacher learn little about the children's complex and dynamic language practices" (García, Wei 2014: 122). No son necesarios grandes cambios. Se trata más bien de modificar la perspectiva y tratar de crear oportunidades que puedan favorecer la apertura y la flexibilidad mental propias de ese "international mindset" que no puede faltar ni en los profesionales de la docencia ni, sobre todo, en el perfil de los actores sociales de mañana.

ARIOLFO R. 2012. Actitudes lingüísticas, inmigración y escuela. Un aporte para la reflexión y la práctica educativa, Lecce, Libellula.

AUGER N. 2014. Exploring the use of migrant languages to support learning in mainstream classrooms in France in C. Leung, D. Little \& P. Van Avermaet (ed.) Managing Diversity in Education, Bristol.

BONOMI M. 2010. Hablamos mitá y mità. Varietà linguistiche di immigrati ispanofoni in Italia, en M.-V. Calvi, G. Mapelli y M. Bonomi [a cura di] Lingua, identità e immigrazione. Prospettive interdisciplinari, Franco Angeli, pp. 53-70.

CALVI M. V. 2015. Cambio de código y conciencia bilingüe en entrevistas a inmigrantes hispanoaericanos en Italia, Revista iberoamericana de linguïstica, Valladolid, Universitas Castellae, pp. 5-31.

CARPANI Daniela, 2008, "Dallo spagnolo all'italiano e ritorno. La lingua immigrata tra strada e scuola" en Carpani Daniela, Capanaga Pilar, De Hériz Ana Lourdes [eds] Convergencias y creatividad, Bologna, Bononia University Press, pp.24-40. 
Hacia una cultura plurilingüe: prácticas educativas en contextos migratorios

D. Carpania
CARPANI D. 2010. Nuovi cittadini, nuove prospettive della scuola interculturale: le ricerche sul campo a Genova, en Lingua, identità e immigrazione. Prospettive interdisciplinari, ob.cit., pp.119-131.

CARAVEDO R. 2010. La percepción en los fenómenos de contacto por migración, Lingua, identità e immigrazione. Prospettive interdisciplinari, ob. cit. pp. 105-117.

CAVALli M., COLETTA D., GAJO L., MATTHEY M. \& SERRA C. 2003, Langues, bilinguisme et représentations sociales au Val d'Aoste - Rapport de recherche, Aoste, IRRE-VDA.

DOSSIER STATISTICO IMMIGRAZIONE. 2015. Roma, Idos.

FAVARO G. 2013. Il bilinguismo disegnato, Italiano LinguaDue, n. 1: 114-127.

GARCÍA O., WEI L. 2014. Language, Bilingualism and Education, New York, Palgrave Macmillan.

VEDOVELLI M. 2004. Italiano e lingue immigrate: comunità alloglotte nelle grandi aree urbane, pp. 587-612 in R. Bombi, F. Fusco (eds.), Città plurilingui. Lingue e culture a confronto in situazioni urbane, Udine, Forum.

\section{NOTES}

Todas las preguntas fueron redactadas en italiano. Aquí para no dificultar la lectura, han sido traducidas al español pasando por alto los errores sintácticos de los textos originales. Por lo que se refiere a la primera encuesta, las dificultades de lectura y escritura han sido obviadas a través del uso masivo de imágenes y de la ayuda de los docentes en la lectura y en la escritura. 